\title{
THE HARDY-LITTLEWOOD THEORENi ON FRACTIONAL INTEGRATION FOR LAGUERRE SERIES
}

\author{
YUICHI KANJIN AND ENJI SATO
}

(Communicated by J. Marshall Ash)

Dedicated to Professor Satoru Igari on his 60th birthday

\begin{abstract}
The Hardy-Littlewood theorem on fractional integration for Fourier series says that if $I_{\sigma} g \sim \sum_{n \neq 0}|n|^{-\sigma} \hat{g}(n) e^{\mathrm{int}}$, then $I_{\sigma}$ is bounded from $L^{p}$ to $L^{q}$, where $1<p<q<\infty, \frac{1}{q}=\frac{1}{p}-\sigma$. We shall establish an analogue of this theorem for the Laguerre function system $\left\{\left(\frac{n !}{\Gamma(n+\alpha+1)}\right)^{\frac{1}{2}} L_{n}^{\alpha}(x) e^{-\frac{x}{2}} x^{\frac{\alpha}{2}}\right\}_{n=0}^{\infty}$.
\end{abstract}

\section{1}

The well-known theorem of Hardy and Littlewood on fractional integration for Fourier series is stated as follows (cf. [Z, Chapter XII]): For $0<\sigma<1$ and a function $g(t)$ on $(0,2 \pi)$, let $I_{\sigma} g \sim \sum_{n \neq 0}|n|^{-\sigma} \hat{g}(n) e^{\text {int }}$, where $\hat{g}(n)$ is the $n$th Fourier coefficient defined by $\hat{g}(n)=\frac{1}{2 \pi} \int_{0}^{2 \pi} g(t) e^{-i n t} d t$. Then, for $g \in L^{p}(0,2 \pi),\left\|I_{\sigma} g\right\|_{q} \leq C\|g\|_{p}, \frac{1}{q}=\frac{1}{p}-\sigma, 1<p, q<\infty$, where $L^{p}(0,2 \pi)$ is the Lebesgue space of all measurable functions $g(t)$ on $(0,2 \pi)$ such that $\|g\|_{p}=\left\{\frac{1}{2 \pi} \int_{0}^{2 \pi}|g(t)|^{p} d t\right\}^{\frac{1}{p}}<\infty$.

The aim of this paper is to establish an analogue of this theorem for Laguerre series by a method transferring boundedness of multiplier operators from Fourier series to Laguerre series.

An earlier result of this kind was obtained for ultraspherical series by Muckenhoupt and Stein [MS, §15] by showing the theorem [MS, Theorem 13] on fractional integration for ultraspherical convolution structure which was first proved by O'Neil [O] in the case of ordinary convolution on a group. They also observed that the same result holds for Hankel transforms. Bavinck [B] proved the Hardy-Littlewood theorem on fractional integration for Jacobi series by using convolution structure. From his result, Gasper and Trebels [GT] derived $(p, q)$-multiplier criterions for Jacobi series. For the harmonic analysis for Hermite and Laguerre expansions, readers may refer to Thangavelu [T].

Let $L_{n}^{\alpha}(x), \alpha>-1$, be the Laguerre polynomial of degree $n$ and of order

Received by the editors November 5, 1993.

1991 Mathematics Subject Classification. Primary 42C10, 42A45.

Key words and phrases. Laguerre polynomials, fractional integration, multipliers, transplantation.

(c) 1995 American Mathematical Society 
$\alpha$ defined by

$$
L_{n}^{\alpha}(x)=\frac{e^{x} x^{-\alpha}}{n !}\left(\frac{d}{d x}\right)^{n}\left(e^{-x} x^{n+\alpha}\right)
$$

and let

$$
\mathscr{L}_{n}^{\alpha}(x)=\sqrt{\frac{\Gamma(n+1)}{\Gamma(n+\alpha+1)}} L_{n}^{\alpha}(x) e^{-\frac{x}{2}} x^{\frac{\alpha}{2}} .
$$

Then the Laguerre function system $\left\{\mathscr{L}_{n}^{\alpha}\right\}_{n=0}^{\infty}$ is complete orthonormal on the interval $(0, \infty)$ with respect to the ordinary Lebesgue measure $d x$. This orthonormal system leads us to the formal expansion of a function $f(x)$ on $(0, \infty)$ :

$$
f \sim \sum_{n=0}^{\infty} a_{n}^{\alpha}(f) \mathscr{L}_{n}^{\alpha}(x),
$$

where $a_{n}^{\alpha}(f)$ is the $n$th Laguerre coefficient of order $\alpha$ of $f(x)$ defined by

$$
a_{n}^{\alpha}(f)=\int_{0}^{\infty} f(x) \mathscr{L}_{n}^{\alpha}(x) d x
$$

We remark that $\left|a_{n}^{\alpha}(f)\right| \leq\|f\|_{p}\left\|\mathscr{L}_{n}^{\alpha}\right\|_{p^{\prime}}, \frac{1}{p}+\frac{1}{p^{\prime}}=1$, and $\left\|\mathscr{L}_{n}^{\alpha}\right\|_{p^{\prime}}<\infty$ if $\alpha \geq 0$ and $1 \leq p \leq \infty$ or if $-1<\alpha<0$ and $\left(1+\frac{\alpha}{2}\right)^{-1}<p \leq \infty$.

For $0<\bar{\sigma}<1$, let $I_{\sigma}^{\alpha}$ be the operator defined by

$$
I_{\sigma}^{\alpha} f \sim \sum_{n=1}^{\infty} \frac{1}{n^{\sigma}} a_{n}^{\alpha}(f) \mathscr{L}_{n}^{\alpha}(x)
$$

for a function $f(x)$ on $(0, \infty)$. We denote by $L^{p}(0, \infty)$ the Lebesgue space of all measurable functions $f(x)$ on $(0, \infty)$ such that $\|f\|_{p}=\left\{\int_{0}^{\infty}|f(x)|^{p} d x\right\}^{\frac{1}{p}}<$ $\infty$. We remark that the $L^{p}(0, \infty)$-norm and the $L^{p}(0,2 \pi)$-norm will be denoted by the same notation. Our theorem is as follows:

Theorem. Let $0<\sigma<1$ and $\alpha>-1$. If $\alpha \geq 0$, then

$$
\left\|I_{\sigma}^{\alpha} f\right\|_{q} \leq C\|f\|_{p}, \quad f \in L^{p}(0, \infty),
$$

for $\frac{1}{q}=\frac{1}{p}-\sigma$ and $1<p, q<\infty$, where $C$ is a constant independent of $f$. If $-1<\alpha<0$, then (1.1) holds for $\frac{1}{q}=\frac{1}{p}-\sigma$ and $\left(1+\frac{\alpha}{2}\right)^{-1}<p, q<-\frac{2}{\alpha}$.

In our proof, we shall use no convolution structure associated with the system $\left\{\mathscr{L}_{n}^{\alpha}\right\}_{n=0}^{\infty}$. Our idea is to prove a transferring theorem (Proposition below) which transfers boundedness of multiplier operators from Fourier series to Laguerre series. We shall derive our theorem from the Hardy-Littlewood theorem for Fourier series and a multiplier criterion for Laguerre series by using the transferring theorem and the Stein complex interpolation theorem.

Let $\Lambda=\left\{\lambda_{n}\right\}_{n=0}^{\infty}$ be a bounded sequence. We define a multiplier operator $F_{\Lambda}^{\alpha}$ for Laguerre series of order $\alpha$ by

$$
F_{\Lambda}^{\alpha} f \sim \sum_{n=0}^{\infty} \lambda_{n} a_{n}^{\alpha}(f) \mathscr{L}_{n}^{\alpha}(x)
$$


Let $1 \leq p, q \leq \infty$. We call $\Lambda$ a $(p, q)$-multiplier for Laguerre series of order $\alpha$ if $\left\|F_{\Lambda}^{\alpha} f\right\|_{q} \leq \bar{C}\|f\|_{p}$ for $f \in L^{p}(0, \infty)$. Also let $\Gamma=\left\{\gamma_{n}\right\}_{n=-\infty}^{\infty}$ be a bounded sequence. We define a multiplier operator $F_{\Gamma}$ for Fourier series

$$
F_{\Gamma} g \sim \sum_{-\infty}^{\infty} \gamma_{n} \hat{g}(n) e^{\mathrm{int}}
$$

We call $\Gamma$ a $(p, q)$-multiplier for Fourier series if $\left\|F_{\Gamma} g\right\|_{q} \leq C\|g\|_{p}$ for $g \in$ $L^{p}(0,2 \pi)$. Then we shall obtain the following proposition.

Proposition. Let $\Gamma=\left\{\gamma_{n}\right\}_{-\infty}^{\infty}$ be a bounded sequence, and define $\Gamma_{+}=\left\{\gamma_{n}\right\}_{n=0}^{\infty}$.

(1) Let $\alpha \geq 0$. Suppose $1<p \leq 2 \leq q<\infty$. If $\Gamma$ is a $(p, q)$-multiplier for Fourier series, then $\Gamma_{+}$is $a(p, q)$-multiplier for Laguerre series of order $\alpha$.

(2) Let $-1<\alpha<0$. If $\left(1+\frac{\alpha}{2}\right)^{-1}<p \leq 2 \leq q<\frac{-2}{\alpha}$, then the assertion of (1) remains true.

The proof of the proposition will be given in the next section. In the rest of this section, we shall show that the proposition implies the theorem.

Let $\alpha>-1$ and $0<\sigma<1$. Also let $p$ and $q$ be a pair of real numbers such that $\frac{1}{q}=\frac{1}{p}-\sigma, 1<p, q<\infty$, when $\alpha \geq 0$, and $\frac{1}{q}=\frac{1}{p}-\sigma,\left(1+\frac{\alpha}{2}\right)^{-1}<$ $p, q<-\frac{2}{\alpha}$, when $-1<\alpha<0$. We can choose $0<t<1,1<p_{0}, q_{0}, p_{1}, q_{1}<$ $\infty, 0<\sigma_{0}<1$ so that $\frac{1}{p}=\frac{t}{p_{0}}+\frac{1-t}{p_{1}}, \frac{1}{q}=\frac{t}{q_{0}}+\frac{1-t}{q_{1}}, \frac{1}{q_{0}}=\frac{1}{p_{0}}-\sigma_{0}$, where $1<p_{0} \leq 2 \leq q_{0}<\infty, 1<p_{1}=q_{1}<\infty$ when $\alpha \geq 0$, and $\left(1+\frac{\alpha}{2}\right)^{-1}<p_{0} \leq$ $2 \leq q_{0}<-\frac{2}{\alpha},\left(1+\frac{\alpha}{2}\right)^{-1}<p_{1}=q_{1}<-\frac{2}{\alpha}$ when $-1<\alpha<0$. We extend the parameter $\sigma$ of the operator $I_{\sigma}^{\alpha}$ to the complex number $z=\sigma+i \theta$. Then we shall show the $\left(L^{p_{0}}, L^{q_{0}}\right)$-boundedness of $I_{\sigma_{0}+i \theta}^{\alpha}$ and the $\left(L^{p_{1}}, L^{q_{1}}\right)$ boundedness of $I_{i \theta}^{\alpha}$. Hence we get the $\left(L^{p}, L^{q}\right)$-boundedness of $I_{\sigma}^{\alpha}$ by using the complex interpolation theorem.

We first note that if $\sigma \geq 0$, then $I_{z}^{\alpha} f \in L^{2}(0, \infty)$ for $f \in L^{2}(0, \infty)$. Let $f$ and $h$ be in $L^{2}(0, \infty)$. Then,

$$
\begin{aligned}
\left|\int_{0}^{\infty} I_{z}^{\alpha} f(x) h(x) d x\right| & =\left|\sum_{n=1}^{\infty} \frac{1}{n^{z}} a_{n}^{\alpha}(f) a_{n}^{\alpha}(h)\right| \\
& \leq \sum_{n=1}^{\infty} \frac{1}{n^{\sigma}}\left|a_{n}^{\alpha}(f)\right|\left|a_{n}^{\alpha}(h)\right| \leq\|f\|_{2}\|h\|_{2} .
\end{aligned}
$$

This implies that the family $\left\{I_{z}^{\alpha}\right\}$ is admissible on the strip $\{z \in \mathbf{C}: 0 \leq \sigma \leq$ $\sigma_{0}$ \}. By applying the multiplier criterion [K, Corollary] (see also [D, Corollary], [T, Theorem 6.3.4], [ST, Corollary 4.4]) for Laguerre series to the multiplier $\left\{n^{-i \theta}\right\}_{n=1}^{n}$, we have

$$
\left\|I_{i \theta}^{\alpha} f\right\|_{q_{1}} \leq C_{\theta}\|f\|_{p_{1}}
$$

for $-\infty<\theta<\infty$, where $C_{\theta}$ is independent of $f$ and admissible growth with respect to $\theta$. Similarly, using the semigroup property $I_{\sigma_{0+i \theta}}^{\alpha}=I_{i \theta}^{\alpha} I_{\sigma_{0}}^{\alpha}$, we have

$$
\left\|I_{\sigma_{0}+i \theta}^{\alpha} f\right\|_{q_{0}} \leq C_{\theta}^{\prime}\left\|I_{\sigma_{0}}^{\alpha} f\right\|_{q_{0}}
$$

for $-\infty<\theta<\infty$, where $C_{\theta}^{\prime}$ has the same property to $C_{\theta}$. The proposition derives the inequality

$$
\left\|I_{\sigma_{0}}^{\alpha} f\right\|_{q_{0}} \leq C\|f\|_{p_{0}}
$$


from the Hardy-Littlewood theorem on fractional integration for Fourier series. Thus, it follows from (1.3) and (1.4) that

$$
\left\|I_{\sigma_{0}+i \theta}^{\alpha} f\right\|_{q_{0}} \leq C_{\theta}^{\prime \prime}\|f\|_{p_{0}}
$$

for $-\infty<\theta<\infty$, where $C_{\theta}^{\prime \prime}$ has the same property to $C_{\theta}$. By the complex interpolation theorem (cf. [SW]), we see that (1.3) and (1.5) lead to

$$
\left\|I_{\sigma}^{\alpha} f\right\|_{q} \leq C\|f\|_{p} \text { for } f \in L^{p}(0, \infty) .
$$

Therefore, the proposition implies the theorem.

In this section, we shall prove the proposition. We use the following transplantation theorem:

Theorem $\mathbf{A}[\mathrm{K}]$. Let $\alpha, \beta>-1$ and $\gamma=\min \{\alpha, \beta\}$. If $\gamma \geq 0$, then

$$
\left\|T_{\alpha}^{\beta} f\right\|_{p} \leq C\|f\|_{p}
$$

for $1<p<\infty$, where $C$ is a constant independent of $f$, and

$$
T_{\alpha}^{\beta} f \sim \sum_{n=0}^{\infty} a_{n}^{\beta}(f) \mathscr{L}_{n}^{\alpha}(x) .
$$

If $-1<\gamma<0$, then (2.1) holds for $\left(1+\frac{\gamma}{2}\right)^{-1}<p<-\frac{2}{\gamma}$.

We note that Theorem A leads to

$$
\left\|T_{\alpha}^{\beta} f\right\|_{p} \sim\|f\|_{p}
$$

since $T_{\alpha}^{\beta} T_{\beta}^{\alpha} f=f$. By virtue of this equivalence, to prove the proposition it is enough to show that

$$
\left\|F_{\Gamma_{+}}^{0} f\right\|_{q} \leq C\|f\|_{p}
$$

for $1<p \leq 2 \leq q<\infty$ if $\Gamma$ is a $(p, q)$-multiplier for Fourier series. For, combining (2.2), (2.3) and the identity $F_{\Gamma_{+}}^{\beta} T_{\beta}^{\alpha} f=T_{\beta}^{\alpha} F_{\Gamma_{+}}^{\alpha} f$, we have $\left\|F_{\Gamma_{+}}^{\alpha} f\right\|_{q} \leq$ $C\left\|T_{0}^{\alpha} F_{\Gamma_{+}}^{\alpha} f\right\|_{q}=C\left\|F_{\Gamma_{+}}^{0} T_{0}^{\alpha} f\right\|_{q} \leq C\left\|T_{0}^{\alpha} f\right\|_{p} \leq C\|f\|_{p}$, where $1<p \leq 2 \leq q<$ $\infty$ if $\alpha \geq 0$, and $\left(1+\frac{\alpha}{2}\right)^{-1}<p \leq 2 \leq q<-\frac{2}{\alpha}$ if $-1<\alpha<0$. Here and below, $C$ denotes a positive constant which may differ at each different occurrence. In order to prove (2.3), we need the following lemma which is a type of transplantation theorem.

Lemma. (1) For a function $g(t)$ on $(0,2 \pi)$, let $U g(x)$ be a function on $(0, \infty)$ defined by the series

$$
U g \sim \sum_{n=0}^{\infty} \hat{g}(n) \mathscr{L}_{n}^{0}(x) .
$$

If $g \in L^{q}(0,2 \pi)$ and $2 \leq q<\infty$, then $U g \in L^{q}(0, \infty)$ and

$$
\|U g\|_{q} \leq C\|g\|_{q} .
$$

(2) For a function $f(x)$ on $(0, \infty)$, let $V f(t)$ be a function defined by the series

$$
V f \sim \sum_{n=0}^{\infty} a_{n}^{0}(f) e^{\mathrm{int}}
$$


If $f \in L^{p}(0, \infty)$ and $1<p \leq 2$, then $V f \in L^{p}(0,2 \pi)$ and

$$
\|V f\|_{p} \leq C\|f\|_{p} .
$$

We easily see that the lemma implies the proposition. Indeed, we have

$$
\left\|F_{\Gamma_{+}}^{0} f\right\|_{q}=\left\|U F_{\Gamma} V f\right\|_{q} \leq C\left\|F_{\Gamma} V f\right\|_{q} \leq C\|V f\|_{p} \leq C\|f\|_{p} .
$$

The first and third inequalities are obtained by (2.4) and (2.5), respectively. The second inequality follows from the assumption that $\Gamma$ is a $(p, q)$-multiplier for Fourier series.

We are now in a position to prove the lemma. Let $C_{c}^{\infty}(0,2 \pi)$ be the space of infinitely differentiable functions with compact support in $(0,2 \pi)$. For $g \in C_{c}^{\infty}(0,2 \pi)$, the sequence $\{\hat{g}(n)\}$ decrease rapidly at infinity. By pointwise and norm estimates for $\mathscr{L}_{n}^{0}(x)$ (cf. [T, Lemmas 1.5.3 and 1.5.4]), we see that for $g \in C_{c}^{\infty}(0,2 \pi)$ the series $\sum_{n=0}^{\infty} \hat{g}(n) \mathscr{L}_{n}^{0}(x)$ converges uniformly and in $L^{p}(0, \infty)$ for every $1 \leq p<\infty$. First we shall show that

$$
U g(x)=\frac{-i}{4 \pi} \int_{0}^{2 \pi} g(t) e^{i \frac{x}{2} \cot \frac{1}{2}} \frac{e^{i \frac{t}{2}}}{\sin \frac{t}{2}} d t, \quad g \in C_{c}^{\infty}(0,2 \pi) .
$$

By the representation [S, (5.4.1)] of Laguerre polynomials in terms of Bessel functions, we have

$$
U g(x)=e^{\frac{x}{2}} \sum_{n=0}^{\infty} \hat{g}(n) \frac{1}{n !} \int_{0}^{\infty} e^{-y} y^{n} J_{0}(2 \sqrt{x y}) d y .
$$

Since the sequence $\{\hat{g}(n)\}$ decreases rapidly at infinity, we can invert the order of summation and integration. Thus, we have

$$
\begin{aligned}
U g(x) & =e^{\frac{x}{2}} \int_{0}^{\infty} e^{-y} J_{0}(2 \sqrt{x y}) \sum_{n=0}^{\infty} \frac{y^{n}}{n !} \hat{g}(n) d y \\
& =\frac{1}{2 \pi} e^{\frac{x}{2}} \int_{0}^{\infty} e^{-y} J_{0}(2 \sqrt{x y}) \sum_{n=0}^{\infty} \int_{0}^{2 \pi} g(t) \frac{\left(y e^{-i t}\right)^{n}}{n !} d t d y .
\end{aligned}
$$

Inverting the order of $\sum_{n=0}^{\infty}$ and $\int_{0}^{2 \pi}$, we have

$$
U g(x)=\frac{1}{2 \pi} e^{\frac{x}{2}} \int_{0}^{\infty} \int_{0}^{2 \pi} J_{0}(2 \sqrt{x} y) g(t) \exp \left(-\left(1-e^{-i t}\right) y\right) d t d y .
$$

We claim that $\int_{0}^{\infty} \int_{0}^{2 \pi}=\int_{0}^{2 \pi} \int_{0}^{\infty}$. Indeed, we denote by $H_{x}(y, t)$ the integrand in the double integral. Since there exists a constant $\varepsilon>0$ such that supp $g \subset$ $[\varepsilon, 2 \pi-\varepsilon]$, it follows that

$$
\left|H_{x}(y, t)\right| \leq\left|J_{0}(2 \sqrt{x y})\right||g(t)| \exp (-(1-\cos \varepsilon) y) .
$$

This inequality leads to our claim. Hence, we have

$$
U g(x)=\frac{1}{2 \pi} e^{\frac{x}{2}} \int_{0}^{2 \pi} g(t) \int_{0}^{\infty} J_{0}(2 \sqrt{x y}) \exp \left(-\left(1-e^{-i t}\right) y\right) d y d t .
$$


It follows from the formula [W, 13.3(1)] that the inner integral has the form

$$
\begin{aligned}
\int_{0}^{\infty} & J_{0}(2 \sqrt{x y}) \exp \left(-\left(1-e^{-i t}\right) y\right) d y \\
\quad= & \int_{0}^{\infty} \exp \left(-\left(1-e^{-i t}\right) \frac{u^{2}}{2}\right) J_{0}(u \sqrt{2 x}) u d u \\
& =\frac{1}{1-e^{-i t}} \exp \left(-\frac{x}{1-e^{-i t}}\right) \quad \text { for } 0<t<2 \pi .
\end{aligned}
$$

Simple calculation shows (2.6).

We shall prove the boundedness of the operator $U$ from $L^{q}(0,2 \pi)$ to $L^{q}(0, \infty)$ for $2 \leq q<\infty$. Let $g \in C_{c}^{\infty}(0,2 \pi)$. By using (2.6) and by changing the variable $u=\frac{1}{2} \cot \frac{t}{2}$, we have

$$
\begin{aligned}
\|U g\|_{q}^{q} & =\pi^{-q} \int_{0}^{\infty}\left|\int_{0}^{2 \pi} g(t) e^{i \frac{x}{2} \cot \frac{1}{2}} \frac{e^{i \frac{t}{2}}}{\sin \frac{t}{2}} d t\right|^{q} d x \\
& =\pi^{-q} \int_{0}^{\infty}\left|\int_{-\infty}^{\infty}\left\{g\left(2 \cot ^{-1} 2 u\right) e^{i \cot ^{-1} 2 u}\left(4 u^{2}+1\right)^{-\frac{1}{2}}\right\} e^{i x u} d u\right|^{q} d x .
\end{aligned}
$$

By the well-known inequality $\int_{-\infty}^{\infty}|\hat{h}(x)|^{q} d x \leq C \int_{-\infty}^{\infty}|h(u)|^{q}|u|^{q-2} d u, 2 \leq$ $q<\infty$, for Fourier transforms (cf. [Ti, Theorem 79]), we have

$$
\begin{aligned}
\|U g\|_{q}^{q} & \leq C \int_{-\infty}^{\infty}\left|g\left(2 \cot ^{-1} 2 u\right)\right|^{q}\left(4 u^{2}+1\right)^{-\frac{q}{2}}|u|^{q-2} d u \\
& \leq C \int_{-\infty}^{\infty}\left|g\left(2 \cot ^{-1} 2 u\right)\right|^{q}\left(4 u^{2}+1\right)^{-1} d u \leq C\|g\|_{q}^{q},
\end{aligned}
$$

which shows $(2.4)$ for $g \in C_{c}^{\infty}(0,2 \pi)$. The standard density argument leads to (1) of the lemma.

We now come to the proof of (2) of the lemma. Let $C_{c}^{\infty}(0, \infty)$ be the space of infinitely differentiable functions with compact support in $(0, \infty)$. Since the sequence $\left\{a_{n}^{0}(f)\right\}$ decreases rapidly at infinity (cf. [K, Lemma 1]), it follows that

$$
\int_{0}^{\infty} f(x) U g(x) d x=\frac{1}{2 \pi} \int_{0}^{2 \pi} V f(t) g(-t) d t
$$

for $f \in C_{c}^{\infty}(0, \infty)$ and $g \in C_{c}^{\infty}(0,2 \pi)$. By a duality argument, we have (2.5) for $f \in C_{c}^{\infty}(0, \infty)$. The density argument concludes the proof of (2) of the lemma.

Remark. The following identity dual to (2.6) can be proved in a very similar way or by a duality argument:

$$
V f(t)=\frac{i e^{-i \frac{t}{2}}}{2 \sin \frac{t}{2}} \int_{0}^{\infty} f(x) e^{-i \frac{x}{2} \cot \frac{t}{2}} d x, \quad f \in C_{c}^{\infty}(0, \infty) .
$$

Note. After submitting this paper, we received a preprint by Gasper, Stempak, and Trebels entitled Fractional integration for Laguerre expansions, which proves the weighted fractional integration theorem using Laguerre convolution. Also, we received a letter from Thangavelu which informed us of a proof of the fractional integration theorem using special Hermite expansions.

We wish to thank Professors G. Gasper, K. Stempak, and W. Trebels for sending their preprint, and Professor S. Thangavelu for sending his letter. 


\section{REFERENCES}

[B] H. Bavinck, A special class of Jacobi series and some applications, J. Math. Anal. Appl. 37 (1972), 767-797.

[D] J. Dlugosz, $L^{p}$-multipliers for the Laguerre expansions, Colloq. Math. 54 (1987), 287-293.

[GT] G. Gasper and W. Trebels, Jacobi and Hankel multipliers of type $(p, q), 1<p<q<\infty$, Math. Ann. 237 (1978), 243-251.

[K] Y. Kanjin, A transplantation theorem for Laguerre series, Tôhoku Math. J. 43 (1991), 537-555.

[MS] B. Muckenhoupt and E. M. Stein, Classical expansions and their relation to conjugate harmonic functions, Trans. Amer. Math. Soc. 118 (1965), 17-92.

[O] R. O'Neil, Convolution operators and L(p,q) spaces, Duke Math. J. 30 (1963), 129-142.

[S] G. Szegö, Orthogonal polynomials, Amer. Math. Soc. Colloq. Publ., Amer. Math. Soc., Providence, RI, 1975.

[ST] K. Stempak and W. Trebels, On weighted transplantation and multipliers for Laguerre expansions, Math. Ann. 300 (1994), 203-219.

[SW] E. M. Stein and G. Weiss, Introduction to Fourier analysis on Euclidean spaces, Princeton Univ. Press, Princeton, NJ, 1971.

[T] S. Thangavelu, Lectures on Hermite and Laguerre expansions, Math. Notes, no. 42, Princeton Univ. Press, Princeton, NJ, 1993.

[Ti] E. C. Titchmarsh, Introduction to the theory of Fourier integrals, Chelsea, New York, 1986.

[W] G. N. Watson, A treaties on the theory of Bessel functions, Cambridge Univ. Press, London, 1966.

[Z] A. Zygmund, Trigonometric series, 2nd ed., Cambridge Univ. Press, London and New York, 1968.

Department of Mathematics, College of Liberal Arts, Kanazawa University, KanaZAWA, 920-11, JAPAN

E-mail address: kanjin€icews1.ipc.kanazawa-u.ac.jp

Department of Mathematics, Faculty of Science, Yamagata University, Yamagata, 990, JAPAN 